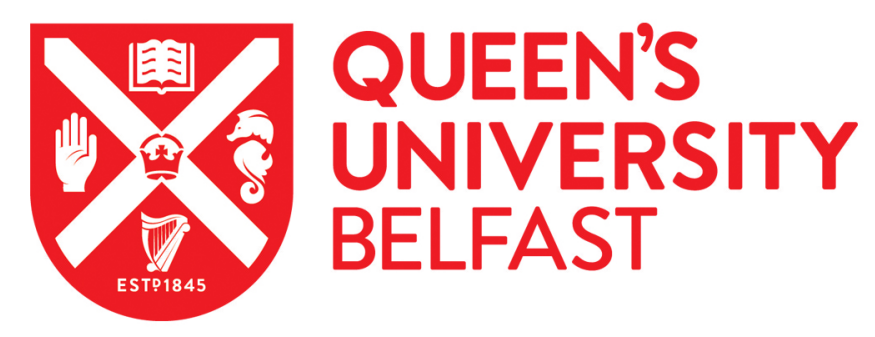

\title{
Evaluation of PTGS2 expression, PIK3CA mutation, aspirin use and colon cancer survival in a population-based cohort study.
}

Gray, R. T., Cantwell, M. M., Coleman, H. G., Loughrey, M. B., Bankhead, P., McQuaid, S., O'Neill, R. F., Arthur, K., Bingham, V., McGready, C., Gavin, A. T., Cardwell, C. R., Johnston, B. T., James, J. A., Hamilton, P. W., Salto-Tellez, M., \& Murray, L. J. (2017). Evaluation of PTGS2 expression, PIK3CA mutation, aspirin use and colon cancer survival in a population-based cohort study. Clinical and translational gastroenterology, 8, [e91]. https://doi.org/10.1038/ctg.2017.18

Published in:

Clinical and translational gastroenterology

Document Version:

Publisher's PDF, also known as Version of record

Queen's University Belfast - Research Portal:

Link to publication record in Queen's University Belfast Research Portal

\section{Publisher rights}

Copyright the authors 2017.

This is an open access article published under a Creative Commons Attribution-NonCommercial-ShareAlike License

(https://creativecommons.org/licenses/by-nc-sa/4.0/), which permits use, distribution and reproduction for non-commercial purposes, provided the author and source are cited and new creations are licensed under the identical terms.

\section{General rights}

Copyright for the publications made accessible via the Queen's University Belfast Research Portal is retained by the author(s) and / or other copyright owners and it is a condition of accessing these publications that users recognise and abide by the legal requirements associated with these rights.

Take down policy

The Research Portal is Queen's institutional repository that provides access to Queen's research output. Every effort has been made to ensure that content in the Research Portal does not infringe any person's rights, or applicable UK laws. If you discover content in the Research Portal that you believe breaches copyright or violates any law, please contact openaccess@qub.ac.uk. 


\section{Evaluation of PTGS2 Expression, PIK3CA Mutation, Aspirin Use and Colon Cancer Survival in a Population-Based Cohort Study}

Ronan T. Gray, MB, BCh (Hons), MSc, MRCS ${ }^{1}$, Marie M. Cantwell, PhD, MPH${ }^{2}$, Helen G. Coleman, PhD', Maurice B. Loughrey, BSc, MRCP, FRCPath, MD ${ }^{3,4}$, Peter Bankhead, BD, MSc, PhD ${ }^{3}$, Stephen McQuaid, PhD, FRCPath ${ }^{3,5}$, Roisin F. O'Neill, $\mathrm{PhD}^{1}$, Kenneth Arthur, MMedSc, FIBMS, $\mathrm{CSci}^{3}$, Victoria Bingham, $\mathrm{MSc}^{3}$, Claire McGready, $\mathrm{BSc}^{3}$, Anna T. Gavin, MB, BCh, MSc, FFPHM ${ }^{6}$, Chris R. Cardwell, PhD ${ }^{1}$, Brian T. Johnston, MD, FRCP ${ }^{7}$, Jacqueline A. James, PhD, FRCPath ${ }^{3,4,5}$, Peter W. Hamilton, BSc, PhD, FRCPath (Hons) ${ }^{3}$, Manuel Salto-Tellez, MD-LMS, FRCPath, FRCP ${ }^{3,4}$ and Liam J. Murray, MD, MRCGP ${ }^{1}$

OBJECTIVES: The association between aspirin use and improved survival after colorectal cancer diagnosis may be more pronounced in tumors that have PIK3CA mutations or high PTGS2 expression. However, the evidence of a difference in association by biomarker status lacks consistency. In this population-based colon cancer cohort study the interaction between these biomarkers, aspirin use, and survival was assessed.

METHODS: The cohort consisted of 740 stage II and III colon cancer patients diagnosed between 2004 and 2008. Aspirin use was determined through clinical note review. Tissue blocks were retrieved to determine immunohistochemical assessment of PTGS2 expression and the presence of PIK3CA mutations. Cox proportional hazards models were used to calculate hazard ratios (HR) and $95 \%$ confidence intervals $(\mathrm{Cl})$ for colorectal cancer-specific and overall survival.

RESULTS: In this cohort aspirin use was associated with a $31 \%$ improvement in cancer-specific survival compared to non-use (adjusted HR $=0.69,95 \% \mathrm{Cl} 0.47-0.98$ ). This effect was more pronounced in tumors with high PTGS2 expression (PTGS2-high adjusted $\mathrm{HR}=0.55,95 \% \mathrm{Cl} 0.32-0.96$ ) compared to those with low PTGS2 expression (PTGS2-low adjusted $\mathrm{HR}=1.19,95 \% \mathrm{Cl}$ 0.68-2.07, P for interaction $=0.09$ ). The aspirin by PTGS2 interaction was significant for overall survival (PTGS2-high adjusted $\mathrm{HR}=0.64,95 \% \mathrm{Cl} 0.42-0.98$ vs. PTGS2-low adjusted $\mathrm{HR}=1.28,95 \% \mathrm{Cl} 0.80-2.03, P$ for interaction $=0.04$ ). However, no interaction was observed between aspirin use and PIK3CA mutation status for colorectal cancer-specific or overall survival.

CONCLUSIONS: Aspirin use was associated with improved survival outcomes in this population-based cohort of colon cancer patients. This association differed according to PTGS2 expression but not PIK3CA mutation status. Limiting adjuvant aspirin trials to PIK3CA-mutant colorectal cancer may be too restrictive.

Clinical and Translational Gastroenterology (2017) 8, e91; doi:10.1038/ctg.2017.18; published online 27 April 2017

Subject Category: Colon/Small Bowel

\section{INTRODUCTION}

Observational studies and long-term follow-up of randomized trials assessing aspirin use in cardiovascular disease indicate that regular aspirin use is associated with a reduced risk of colorectal neoplasia. ${ }^{1}$ Further long-term follow-up of these trials suggests aspirin use is associated with a reduction in both the incidence and subsequent development of metastatic disease. ${ }^{2}$ Similarly, a recent population-based cohort study with over 23,000 colorectal cancer patients demonstrated that post-diagnostic aspirin use was associated with improved cancer-specific survival. ${ }^{3}$ Pre-clinical evidence confirms the biological plausibility of these associations and suggests aspirin may have pleiotropic anti-cancer effects. ${ }^{4}$ Although the exact mechanisms remain unclear they are likely to involve prostaglandin-endoperoxide synthase 2 (PTGS2, also known as cyclooxygenase-2 or COX-2) dependent and independent pathways. ${ }^{4,5}$ Taken together, this data suggests aspirin could have utility in the adjuvant treatment of colorectal cancer.

A number of clinical trials assessing the role of adjuvant aspirin therapy (low- and high-dose) in colorectal cancer have recently started recruitment (NCT00565708, NCT02607072, NCT02301286, ISRCTN74358648). However, the potential side effects associated with aspirin use, including major gastrointestinal bleeding, and the understanding that colorectal cancers are heterogeneous has led to attempts to better

\footnotetext{
${ }^{1}$ Cancer Epidemiology and Health Services Research Group, Centre for Public Health, Queen's University Belfast, Belfast, Northern Ireland; ${ }^{2}$ Nutrition and Metabolism Group, Institute for Global Food Security, Queen's University Belfast, Belfast, Northern Ireland; ${ }^{3}$ Northern Ireland Molecular Pathology Laboratory, Centre for Cancer Research and Cell Biology, Queen's University Belfast, Belfast, Northern Ireland; ${ }^{4}$ Tissue Pathology, Belfast Health and Social Care Trust, Belfast, Northern Ireland; ${ }^{5}$ Northern Ireland Biobank, Queen's University Belfast, Belfast, Northern Ireland; ${ }^{6}$ Northern Ireland Cancer Registry, Queen's University Belfast, Belfast, Northern Ireland and ${ }^{7}$ Department of Gastroenterology, Belfast Health and Social Care Trust, Belfast, Northern Ireland

Correspondence: R.T. Gray, MB BCh (Hons), MSc, MRCS, Cancer Epidemiology and Health Services Research Group, Centre for Public Health, Queen's University Belfast, Royal Victoria Hospital, Belfast BT12 6BA, Northern Ireland. E-mail: rgray05@qub.ac.uk

Received 9 January 2017; accepted 14 March 2017
} 
identify those patients who may derive a survival benefit. ${ }^{6,7}$ Allowing for tumor heterogeneity, molecular pathological epidemiology studies can facilitate assessment of the interaction between molecular biomarkers and lifestyle or other exogenous factors and the subsequent effect on cancer survival. ${ }^{5,8}$ As a result, they can provide mechanistic insight into the anti-cancer effect of the exposure being assessed and contribute to the development of precision medicine. $^{5}$

PTGS2 is the rate limiting enzyme in the conversion of arachidonic acid to prostaglandins. ${ }^{9}$ Prostaglandins have the potential to enhance the neoplastic process by inhibiting apoptosis, stimulating proliferation and promoting angiogenesis. ${ }^{4}$ Phosphatidylinositol 3-kinase (PI3K) signaling pathways also play an important role in colorectal cancer carcinogenesis. $^{6} \quad$ Phosphatidylinositol-4,5-biphosphonate 3-kinase (PIK3CA) encodes the p110a catalytic subunit of $\mathrm{PI} 3 \mathrm{~K}$ and mutations in this gene constitutively activate the PI3K pathway. 7,10 Subsequent downstream activation of protein kinase $B(A K T)$ results in PTGS2 upregulation and the potential to inhibit apoptosis. ${ }^{11,12}$ Overexpression of PTGS2 and the presence of PIK3CA mutations have been shown to differentiate the survival benefit associated with aspirin use in some $6,7,9$ but not all ${ }^{7,13,14}$ colorectal cancer observational studies. As the evidence of a difference in association by biomarker status lacks consistency further validation is required.

Therefore, the aim of this study was to assess the interaction between aspirin use, the biomarkers PTGS2 and PIK3CA, and survival in a population-based cohort study of patients with stage II and III colon cancer.

\section{METHODS}

Study cohort. All stage II and III colon cancer patients (International Classification of Disease code C18) diagnosed in Northern Ireland between 2004 and 2008 were identified from the Northern Ireland Cancer Registry $(n=1,862)$. Rectal cancers were excluded from this molecular pathological epidemiology study due to the potential for neoadjuvant radiotherapy to alter tumor expression profiles. Following review of pathology reports the cohort was restricted to patients with a single primary colon adenocarcinoma who had undergone a surgical resection $(n=1,539)$. A further 113 patients were excluded after clinical note review for reasons listed in Figure 1. To facilitate formalin fixed, paraffinembedded (FFPE) tissue block retrieval the final cohort was restricted to patients from two of the five Northern Ireland Health and Social Care trusts within the remit of the Northern Ireland Biobank (740 of 1,426, 51.9\%). These patients were representative of the overall Northern Ireland cohort in terms of age, sex, tumor stage, adjuvant chemotherapy use and proportion of deaths that occurred (see Supplementary Table S1 online).

Clinicopathological variables and follow-up. Clinical variables including adjuvant chemotherapy use, prescription medication use, family history of colorectal cancer and Eastern Cooperative Oncology Group (ECOG) performance status were retrieved from the Northern Ireland Clinical Oncology Information System (COIS), a prospective electronic record of cancer patient management. This process was supplemented by a manual chart review when insufficient information was recorded on COIS or when patients were not referred to an oncologist for consideration of adjuvant therapy. Stage and grade of differentiation were extracted from pathology reports. All patients were followed up for occurrence and cause of death via linkage to the Northern Ireland Registrar General's Office up to 31st December 2013. Colorectal cancer-specific deaths were defined as those with an underlying cause of death International Classification of Disease code C18, C19, C20 (anus) and/or C26 (other and ill-defined digestive organs).

Assessment of aspirin use. Prescription aspirin use (user vs. non-user) was assessed early in the post-operative period for all patients. When medication information was available on COIS this time point was the initial post-operative oncology review where current regularly prescribed medication use is recorded. When medication information was not available on COIS aspirin exposure was determined from the post-operative hospital discharge letter. Information on medication dosage was not consistently recorded on COIS. Our research group has previously demonstrated that $98.5 \%$ of aspirin prescriptions after colorectal cancer diagnosis in the UK are for low-dose $(75 \mathrm{mg})$ aspirin. ${ }^{15}$ Exposure in this study is therefore considered representative of low-dose aspirin.

\section{Tumor molecular analysis}

FFPE block retrieval and tissue microarray construction. The Northern Ireland Biobank retrieved slides from all of the resection specimen blocks for each case. These original hematoxylin and eosin-stained slides were reviewed by an expert gastrointestinal histopathologist to select the two most representative FFPE tumor-rich blocks. Following block retrieval, a new section was cut for hematoxylin and eosin staining and the slides were annotated for tissue microarray (TMA) construction. Blocks were retrieved for $89.3 \%$ of the cohort (661 out of 740). Three representative areas within the donor blocks of each case were annotated for targeted coring. Three $1.0 \mathrm{~mm}$ diameter tissue cores were then extracted from donor blocks and inserted into recipient blocks using a manual tissue arrayer (Estigen, Tartu, Estonia) as described previously. ${ }^{16,17}$

PTGS2 immunohistochemistry, scoring and assessment. All immunohistochemistry was performed in the Northern Ireland Molecular Pathology Laboratory, a research laboratory which has UK Clinical Pathology Accreditation. In brief, all immunohistochemistry was performed on the fully automated Ventana BenchMark XT platform using an OptiView antibody detection system (Ventana Medical Systems Inc., Tucson, USA) and a Cell Marque ready to use rabbit monoclonal antibody to PTGS2 (Ventana, catalog number 760-4254). QuPath (Queen's University Belfast, Northern Ireland) image analysis software facilitated digital immunoscoring. More detailed information is provided in the Supplementary Methods (online). A H-score was calculated based on the extent and intensity of cytoplasmic staining $(3 \times \%$ of 


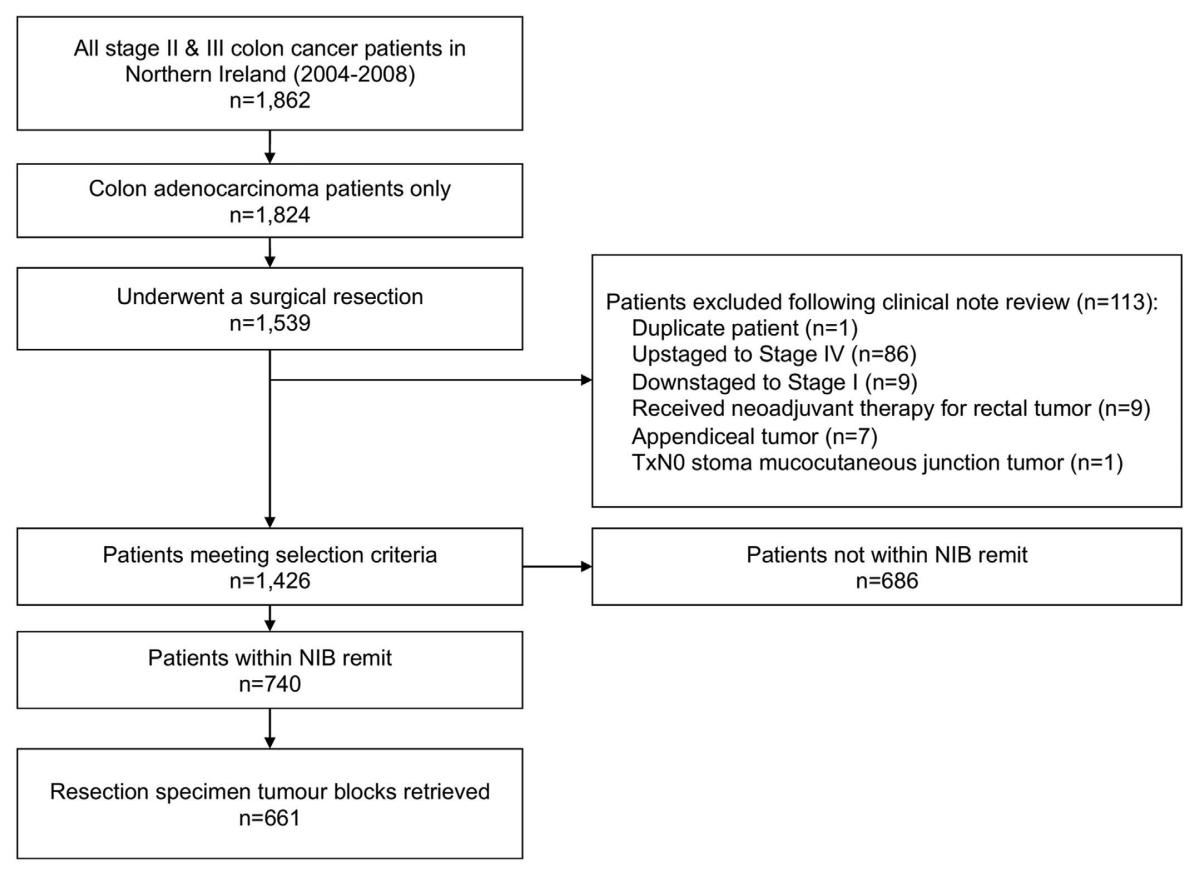

Figure 1 Selection of stage II and III colon cancer (adenocarcinoma) patients and samples. NIB, Northern Ireland Biobank.

strongly staining cytoplasm $+2 \times \%$ of moderately staining cytoplasm $+1 \times \%$ of weakly staining cytoplasm, giving a range of $0-300) .^{18}$

Independently, an expert gastrointestinal histopathologist assessed PTGS2 expression in a random 10\% sample (207 of the triplicate TMA cores) using the standardized grading system (absent, weak, moderate or strong immunostaining) described by Chan et al. ${ }^{9}$ Tumors with low PTGS2 expression (PTGS2-low) were categorized as those demonstrating weak or absent immunostaining while PTGS2-high tumors had moderate or strong levels of immunostaining. Receiveroperating characteristic analysis was subsequently used to determine the optimal $\mathrm{H}$-score cutoff point that predicted PTGS2 grading with maximal sensitivity and specificity (according to Youden's J statistic using Cutoff Finder ${ }^{19}$ ). Using this methodology, a H-score of 145.5 differentiated PTGS2low from PTGS2-high tumors with good interobserver agreement between the digital $\mathrm{H}$-score and the histopathologist's grading (agreement $86.5 \%, \kappa=0.70$ ). In the final data set, PTGS2 expression grading was determined using the median $\mathrm{H}$-score from the three cores available for each case. Values above and below 145.5 were considered PTGS2-high and PTGS2-low respectively. Representative images are shown in Figure 2.

DNA extraction. Tumor-rich areas of the representative blocks from each case were annotated for macrodissection. DNA was extracted according to the manufacturer's instructions from five $5 \mu \mathrm{m}$ sections using the Maxwell 16 Instrument (Promega, Southampton, UK) and Promega DNA extraction kit. Quantification of DNA was performed by an absorbance method using the NanoDrop 2000c spectrophotometer (Thermo Scientific, Wilmington, USA).
PIK3CA mutation and microsatellite instability status. Mass spectrometry incorporating the pre-validated ColoCarta Panel (Agena Bioscience, Hamburg, Germany) was used to detect the presence of PIK3CA mutations. Samples were processed at the Genomics Core Technology Unit (Queen's University Belfast, Northern Ireland) and the Assays by Agena Custom Services Laboratory (Hamburg, Germany) using previously described methods. ${ }^{20}$ Detection of somatic mutations at a frequency of $>10 \%$ for any of eight alleles (C420R, E542K, E545K, H701P, H1047R, H1047L, Q546K, or $\mathrm{R} 88 \mathrm{Q}$ ) was considered evidence of a mutation in the PIK3CA gene. A failed reaction at a single position resulted in missing data for PIK3CA status only if the reactions at other positions were wild-type. ${ }^{21}$ This panel includes assays that capture $79 \%$ of known PIK3CA mutations including the 'hot spot' mutations in exons 9 and 20 (Q546K, E545K, E542K, H1047R, and H1047L), which confer constitutive kinase activity. $^{10}$

Microsatellite instability (MSI) analysis was performed within the Northern Ireland Molecular Pathology Laboratory according to manufacturer's instructions using the MSI Analysis System, version 1.2 kit (Promega, Southampton, UK) for five mononucleotide repeat markers (BAT-25, BAT-26, NR-21, NR-24 and MONO-27). PCR products were separated by capillary electrophoresis using an ABI 3500 Genetic Analyzer (Fisher Scientific-UK Ltd, Loughborough, UK). The output data were analyzed using GeneMapper v4.1 (Fisher Scientific-UK Ltd, Loughborough, UK) to determine MSI-high (MSI-H) status (MSI-H vs. non MSI-H).

Statistical analysis. All statistical analysis was performed using Stata 14 (StataCorp, College Station, TX, USA). The $x^{2}$ test was used to compare characteristics between aspirin 
a

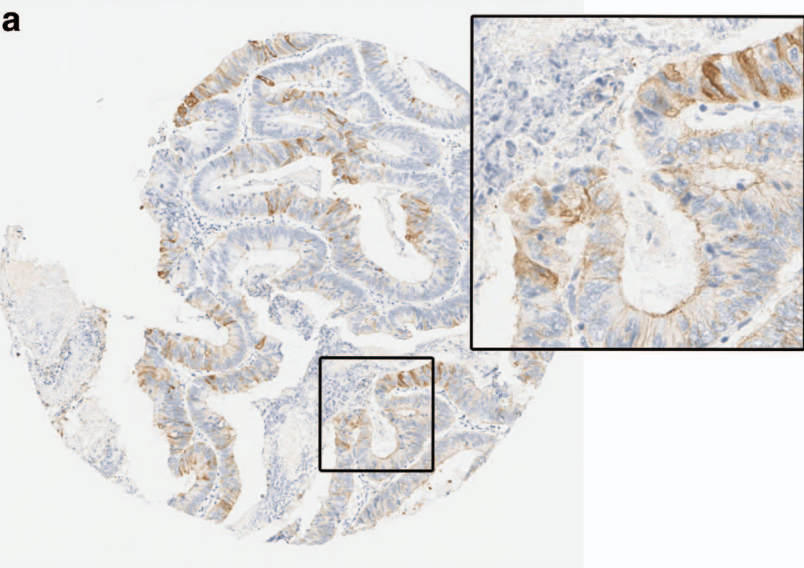

C

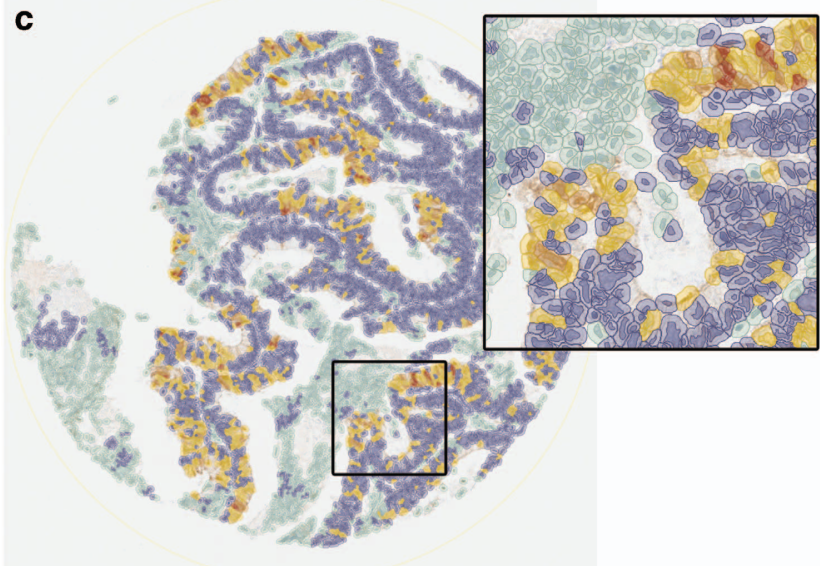

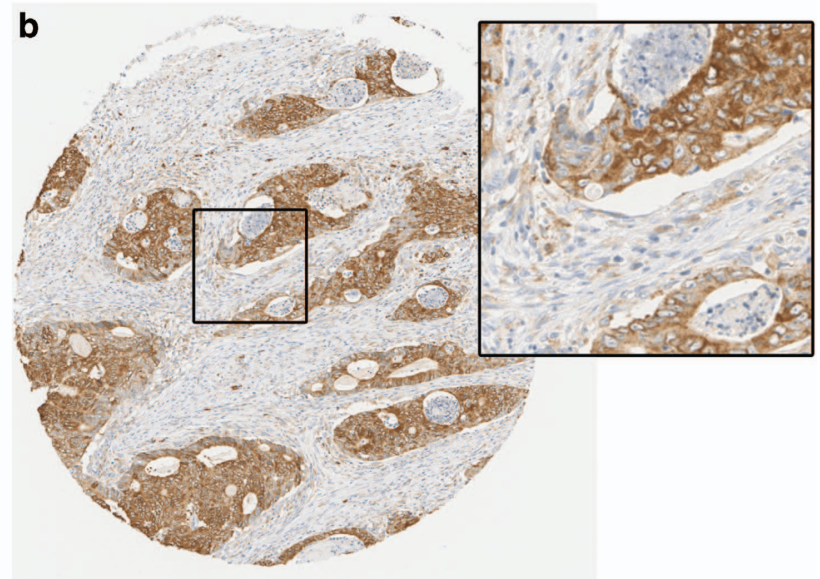

d

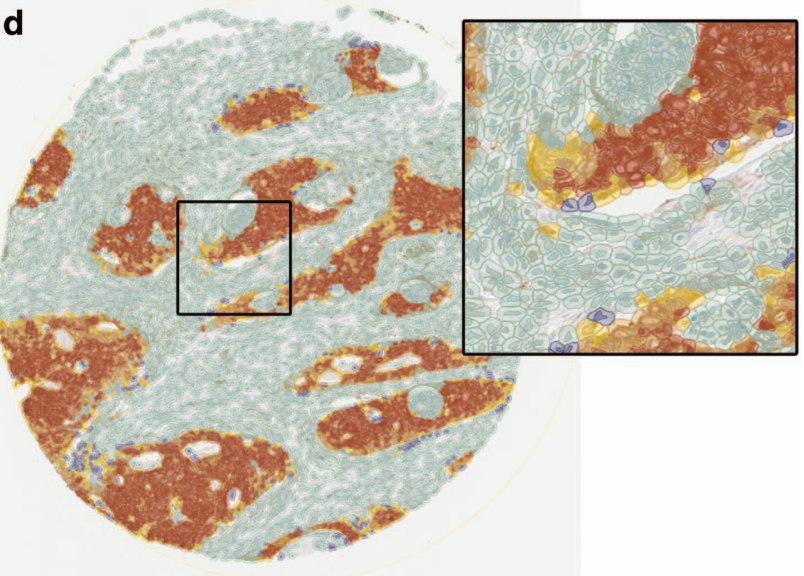

Figure 2 PTGS2 immunohistochemistry in colon cancer tissue microarrays (TMAs) and associated mark-up for digital immunoscoring using QuPath image analysis software. Detected cells are color-coded according to their classification: green, non-tumor; blue, negatively staining tumor; yellow, weakly staining tumor; orange, moderately staining tumor; red, strongly staining tumor. (a) Original core from a tumor weakly expressing PTGS2 (PTGS2-low). (b) Original core from a tumor strongly expressing PTGS2 (PTGS2-high). (c) QuPath cellular mark-up in the PTGS2-low core (H-score 30.3). (d) QuPath cellular mark-up in the PTGS2-high core (H-score 243.4).

users and non-users. The primary outcome of this study was colorectal cancer-specific survival and the secondary outcome was overall survival. The association between aspirin use and survival was assessed in the whole cohort and then in analyses stratified by biomarker status. Only cases with information on aspirin exposure (known user vs. known nonuser) were included in the former analysis. Only cases with available exposure information and tissue for biomarker assessment were included in the subsequent stratified analyses. Other missing categorical data were coded as unknown.

Survival analysis was performed using the Cox proportional hazards model to calculate hazard ratios (HRs) and associated $95 \%$ confidence intervals $(\mathrm{Cl})$. To control for confounding the cancer-specific survival multivariable model adjusted for age category at diagnosis $(<50 / 50-<60 / 60-<70 / 70$ $<80 />80$ ), gender, year of diagnosis (continuous variable), stage (II/III), grade (well or moderate/poor/unknown), MSI status (MSI-H/non MSI-H/unknown), adjuvant chemotherapy use within three months of surgery (yes/no), ECOG performance status (0-1/2/3-4/unknown) and family history of colorectal cancer (yes/no/unknown). In addition to the above variables, the overall survival multivariable model also adjusted for the Charlson Comorbidity Index score (continuous variable). ${ }^{22}$ Analyses were stratified by biomarker status. Interaction terms for aspirin and PIK3CA or PTGS2 were then included in the Cox model and the Wald test was used to assess for statistical interaction. Sensitivity analyses adjusted for statin use and limited PIK3CA mutations to exons 9 and 20 mutations (confer constitutive kinase activity). Sensitivity analyses were also conducted based on altering the PTGS2 $\mathrm{H}$-score cutoff point. All $P$ values were two-sided and a value $<0.05$ was considered statistically significant.

Ethics. Ethical approval was obtained and tissue was acquired through the Northern Ireland Biobank (NIB ref. 13-0088) under the remit of the biobank's ethical approval from the Office of Research Ethics Committees Northern Ireland (ORECNI ref. 16/NI/0030).

\section{RESULTS}

Patients. Of the 740 patients identified in this populationbased cohort study, information on prescription medication use 
Table 1 Clinical and demographic characteristics according to aspirin use

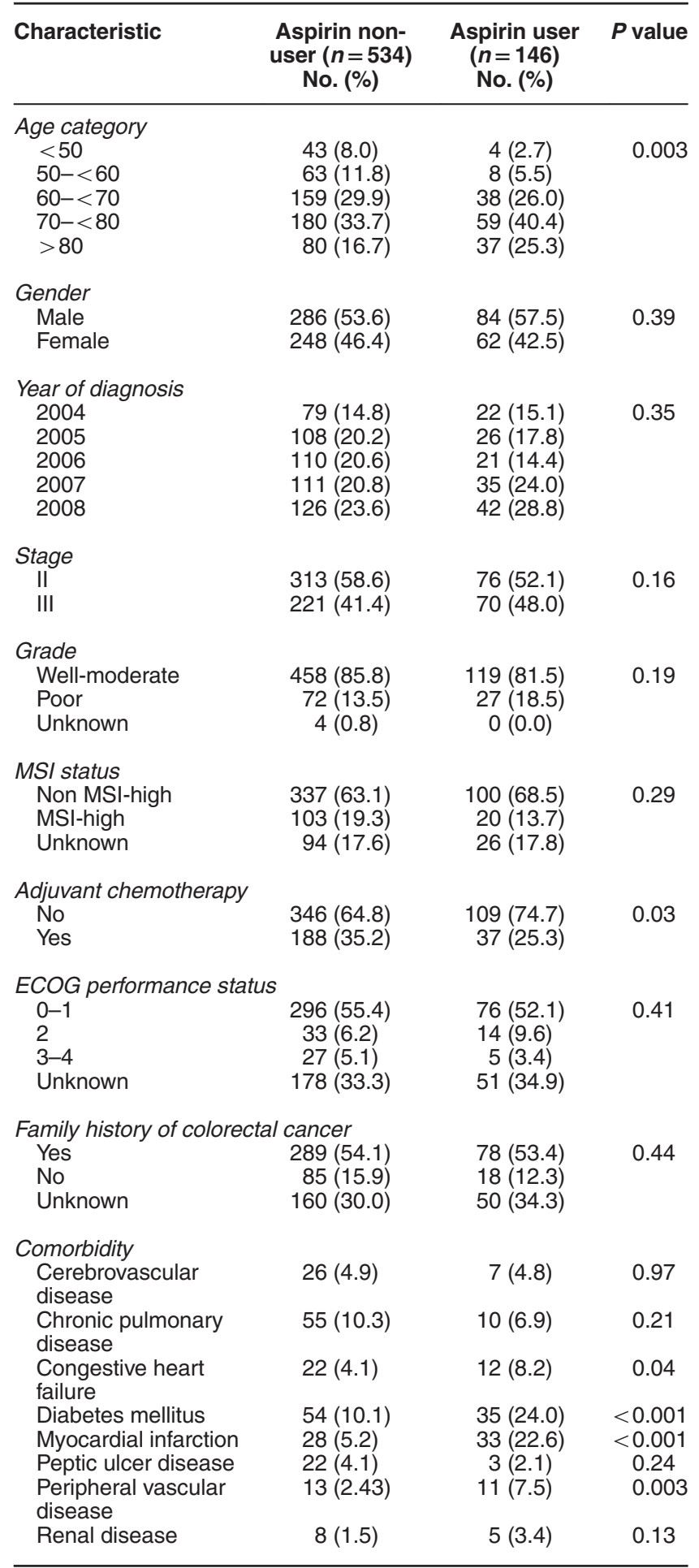

ECOG, Eastern Cooperative Oncology Group; MSI, microsatellite instability.

in the early post-operative period was available for 680 $(91.9 \%)$. Compared to those with available information on medication use, patients with no information on medication use were older and more likely to be diagnosed in the earlier years

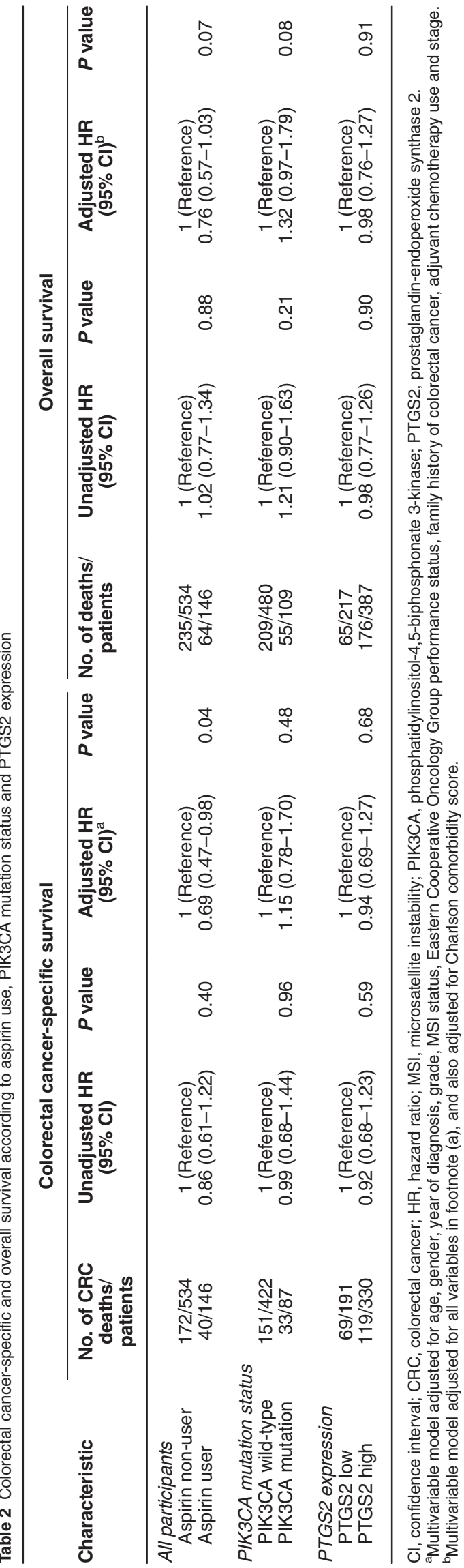


of the study. However, there was no difference in stage, grade of tumor differentiation, or MSI status (see Supplementary Table S2). In patients with known information on medication use, there were $146(21.5 \%)$ aspirin users. After a mean followup of 5.7 (range 0-10) years there were 299 all-cause and 212 colorectal cancer-specific deaths among these patients.

Aspirin use and survival. Table 1 summarizes the baseline characteristics between aspirin users and non-users. Compared to aspirin non-users, aspirin users were older and less likely to receive adjuvant chemotherapy. As expected aspirin users also had significantly more cardiovascular disease and diabetes. However, tumor grade, stage, and MSI status were similar between users and non-users. Aspirin use at the time of diagnosis was associated with a $31 \%$ reduction in colorectal cancer-specific mortality (adjusted $\mathrm{HR}=0.69,95 \% \mathrm{Cl} 0.47-0.98$ ) compared to nonuse (Table 2). The association between aspirin use and overall mortality was slightly attenuated (adjusted $\mathrm{HR}=0.76$, 95\% Cl 0.57-1.03).

PIK3CA mutations. DNA was extracted in 599 tumor blocks of the 680 patients in the cohort with information on medication use at the time of diagnosis. PIK3CA mutation status (mutant vs. wild type) was available for $98.3 \%$ of these samples (589 out of 599). There was no difference in the proportion of aspirin users in patients with extracted DNA compared to those without extracted DNA (21.5 vs. $21.0 \%$ ). There was a lower proportion of
PIK3CA-mutant tumors in aspirin users (17 out of 129 , $13.2 \%$ ) compared to aspirin non-users (92 of $460,20.0 \%$ ) although the difference was not statistically significant $(P=0.08)$.

Compared to wild-type PIK3CA, the presence of a PIK3CA mutation was not significantly associated with colorectal cancer-specific survival (adjusted $\mathrm{HR}=1.15,95 \% \mathrm{Cl} 0.78-$ 1.70, Table 2). In stratified analysis there was also no evidence that the association between aspirin use and colorectal cancer-specific survival differed by PIK3CA mutation status (mutant PIK3CA adjusted $\mathrm{HR}=0.66,95 \% \mathrm{Cl}$ $0.22-2.01$ vs. wild-type PIK3CA adjusted $\mathrm{HR}=0.69,95 \% \mathrm{Cl}$ $0.46-1.05, P$ for interaction 0.80$)$. Similar results were observed for overall survival (Table 3 ).

PTGS2 immunohistochemical expression. TMAs were created from 604 tumor blocks where matched information on medication use was available. There were similar proportions of aspirin users in cases with matched TMA data compared to those without matched TMA data (21.5 vs. $21.1 \%)$. The proportion of PTGS2-high tumors was $57.7 \%$ (75 of 130) among aspirin users and 65.8\% (312 of 474$)$ among aspirin non-users $(P=0.09)$. PTGS2 expression was not associated with colorectal cancer-specific survival when PTGS2-high tumors were compared to PTGS2-low tumors (adjusted $\mathrm{HR}=0.94,95 \% \mathrm{Cl}$ 0.69-1.27, Table 2).

In analyses stratified by PTGS2 expression, aspirin users had better colorectal cancer-specific survival compared to

Table 3 Aspirin use and colorectal cancer-specific and overall survival stratified by PIK3CA mutation status and PTGS2 immunohistochemical expression

\begin{tabular}{|c|c|c|c|c|c|c|}
\hline & \multicolumn{2}{|c|}{ Colorectal cancer-specific survival $^{\mathrm{a}}$} & \multirow[t]{2}{*}{$P$ value } & \multicolumn{2}{|c|}{ Overall survival $^{\mathrm{b}}$} & \multirow[t]{2}{*}{$P$ value } \\
\hline & Aspirin non-user & Aspirin user & & Aspirin non-user & Aspirin user & \\
\hline \multicolumn{7}{|l|}{ PIK3CA mutation } \\
\hline No. of deaths/patients & $28 / 92$ & $5 / 17$ & & $46 / 92$ & $9 / 17$ & \\
\hline Unadjusted HR $(95 \% \mathrm{Cl})$ & 1 (Reference) & $1.03(0.40-2.67)$ & 0.96 & 1 (Reference) & $1.22(0.59-2.50)$ & 0.59 \\
\hline Adjusted HR $(95 \% \mathrm{Cl})$ & 1 (Reference) & $0.66(0.22-2.01)$ & 0.47 & 1 (Reference) & $0.79(0.35-1.78)$ & 0.57 \\
\hline \multicolumn{7}{|l|}{ PIKЗCA wild-type } \\
\hline No. of deaths/patients & $120 / 368$ & $31 / 112$ & & $159 / 368$ & $50 / 112$ & \\
\hline Unadjusted HR (95\% Cl) & 1 (Reference) & $0.87(0.58-1.30)$ & 0.50 & 1 (Reference) & $1.07(0.78-1.48)$ & 0.67 \\
\hline Adjusted HR $(95 \% \mathrm{Cl})$ & 1 (Reference) & $0.69(0.46-1.05)$ & 0.09 & 1 (Reference) & $0.80(0.57-1.13)$ & 0.20 \\
\hline Aspirin by PIK3CA interaction & - & - & 0.80 & - & - & - \\
\hline \multicolumn{7}{|l|}{ PTGS2-high } \\
\hline No. of deaths/patients & $102 / 312$ & $17 / 75$ & & $146 / 312$ & $30 / 75$ & \\
\hline Unadjusted HR (95\% Cl) & 1 (Reference) & $0.66(0.39-1.12)$ & 0.12 & 1 (Reference) & $0.83(0.56-1.24)$ & 0.37 \\
\hline Adjusted HR $(95 \% \mathrm{Cl})$ & 1 (Reference) & $0.55(0.32-0.96)$ & 0.04 & 1 (Reference) & $0.64(0.42-0.98)$ & 0.04 \\
\hline \multicolumn{7}{|l|}{ PTGS2-Iow } \\
\hline No. of deaths/patients & $50 / 162$ & $19 / 55$ & & $66 / 162$ & $29 / 55$ & \\
\hline Unadjusted HR (95\% Cl) & 1 (Reference) & $1.25(0.73-2.12)$ & 0.41 & 1 (Reference) & $1.45(0.94-2.25)$ & 0.09 \\
\hline Adjusted HR (95\% Cl) & 1 (Reference) & $1.19(0.68-2.07)$ & 0.55 & 1 (Reference) & $1.28(0.80-2.03)$ & 0.30 \\
\hline Aspirin by PTGS2 interaction & - & - & 0.09 & - & - & 0.04 \\
\hline
\end{tabular}

$\mathrm{CI}$, confidence interval; HR, hazard ratio; MSI, microsatellite instability; PIK3CA, phosphatidylinositol-4,5-biphosphonate 3-kinase; PTGS2, prostaglandinendoperoxide synthase 2 .

aMultivariable model adjusted for age, gender, year of diagnosis, grade, MSI status, Eastern Cooperative Oncology Group performance status, family history of colorectal cancer, adjuvant chemotherapy use and stage.

${ }^{\mathrm{b}}$ Multivariable model adjusted for all variables in footnote (a), and also adjusted for Charlson comorbidity score. 
non-users in PTGS2-high tumors (adjusted $\mathrm{HR}=0.55,95 \% \mathrm{Cl}$ 0.32-0.96), whereas no improvement was observed in PTGS2-low tumors (adjusted $\mathrm{HR}=1.19,95 \% \mathrm{Cl} 0.68-2.07$, $P$ for interaction 0.09, Table 3). Similar results were observed for overall survival with evidence of a significant interaction (PTGS2-high adjusted $\mathrm{HR}=0.64,95 \% \mathrm{Cl} 0.42-0.98$ vs. PTGS2-low adjusted $\mathrm{HR}=1.28,95 \% \mathrm{Cl} 0.80-2.03, P$ for interaction $=0.04$, Table 3).

Sensitivity/subgroup analyses. The associations described above were not markedly altered when statin use at the time of diagnosis was included in the multivariable model (Table 4), or when PIK3CA mutations were limited to exons 9 and 20 (see Supplementary Table S3). However, the association between improved cancer-specific survival and aspirin use in PTGS2-high tumors was more pronounced when the PTGS2 $\mathrm{H}$-score cutoff point was increased. This association was attenuated when the cutoff point was decreased (see Supplementary Table S4).

\section{DISCUSSION}

Exposure to aspirin was associated with improved survival in this population-based cohort study of stage II and III colon cancer patients. In stratified analyses there was no evidence of an interaction between aspirin use and tumor PIK3CA mutation status. However, the association between aspirin use and improved survival was more pronounced in tumors with higher levels of PTGS2 expression.

Previous studies investigating the association between these biomarkers (PTGS2 and PIK3CA), aspirin use and survival in colorectal cancer have reported mixed results. Using data from two US cohort studies, Chan et al. ${ }^{9}$ demonstrated that aspirin use after colorectal cancer diagnosis was associated with marked survival benefits in tumors that overexpressed PTGS2 compared to those that did not. Reimers et al. ${ }^{14}$ were unable to replicate this finding in a large Dutch population-based cohort study. Similarly, Domingo et al. ${ }^{7}$ did not observe a differential survival effect for aspirin use according to PTGS2 status following molecular analysis of the cohort of patients enrolled in the VICTOR trial.

The first notable difference between these studies is that Chan et al. ${ }^{9}$ considered both high- and low-dose aspirin users whereas the other studies were limited to low-dose aspirin only. The proportion of PTGS2-positive tumors in these cohorts also varied considerably $\left(68.4 \%^{9}\right.$ vs. $56.3 \%{ }^{14}$ vs. $21.0 \%{ }^{7}$ respectively). In addition to the choice of primary antibody, differences in the definition of PTGS2 overexpression were also present. In particular, while all studies have assessed the intensity of PTGS2 immunostaining using the methods described by Chan et al. ${ }^{9}$ only tumors with the strongest staining intensity were considered overexpressors by Domingo et al. ${ }^{7}$ Subsequently there was a marked discrepancy between the proportions of PTGS2-positive tumors (21.0 vs. $68.4 \%$ ) in the VICTOR trial ${ }^{7}$ cohort and the seminal US cohorts. ${ }^{9}$

The current population-based cohort study is the first to corroborate the findings described by Chan et al. ${ }^{9}$ 
regarding an interaction between aspirin exposure, PTGS2 overexpression and better survival in colorectal cancer. Similar to the Dutch ${ }^{14}$ and VICTOR trial ${ }^{7}$ cohorts, the current study is representative of low-dose aspirin exposure only. Importantly though, $64.1 \%$ of tumors were defined as having higher levels of PTGS2 expression in our study, which more closely matches the proportions observed using the methods described by Chan et al. ${ }^{9}$ This association also became more pronounced in sensitivity analyses that increased the PTGS2 $\mathrm{H}$-score threshold, which increases the robustness and biological plausibility of this finding.

Given the problems associated with standardizing immunohistochemical techniques across pathology laboratories, mutated PIK3CA has been proposed as a more reliable biomarker to identify colorectal tumors sensitive to aspirin. ${ }^{6}$ Liao et al. $^{6}$ were the first to report that the presence of PIK3CA mutations differentiated the improved survival benefit observed with aspirin use in colorectal cancer. This finding was subsequently validated using data from the VICTOR trial cohort. ${ }^{7}$ Similar to the findings in the Dutch colorectal cancer cohort ${ }^{14}$ however, we did not observe evidence of an interaction between low-dose aspirin use and the presence of PIK3CA mutations. Aspirin use at the time of diagnosis was also not associated with a significant improvement in colorectal cancer-specific survival in a retrospective cohort of 185 patients with mutant PIK3CA tumors. ${ }^{13}$ However, stage IV tumors were included in that cohort and there was no comparative group to assess for an interaction.

Two meta-analyses have assessed the interaction between aspirin use after diagnosis and PIK3CA mutation in observational studies of colorectal cancer patients. ${ }^{23,24}$ Paleari et al. ${ }^{23}$ found that aspirin use in patients with PIK3CA mutated tumors was associated with a $29 \%$ significant reduction in overall mortality. ${ }^{23}$ In the meta-regression analysis, however, there was no evidence of a significant interaction between PIK3CA mutation status and aspirin efficacy $(P=0.40)$ for overall survival. The authors of this review did, however, recognize the potential for immortal time bias in two of the included studies assessing overall survival, ${ }^{6,7}$ where follow-up commenced at the time of colorectal cancer diagnosis but exposure to aspirin treatment started at a non-specified time during subsequent followup. $^{23,25}$ In the present study aspirin use was assessed early in the post-operative period therefore the risk of immortal time bias is minimized.

A more recent meta-analysis by Elwood et al. ${ }^{24}$ has also noted a marked protective association for aspirin and colorectal cancer-specific survival in PIK3CA-mutant tumors. However, the magnitude of their risk estimate $(\mathrm{HR}=0.45,95 \% \mathrm{Cl} 0.28-0.71)$ may be exaggerated due to the inclusion of an estimate for recurrence-free survival from one study that did not report cancer-specific survival ${ }^{7}$ and erroneous inclusion of an estimate relating to PTGS2-high tumors, rather than PIK3CA-mutant tumors, from another study. ${ }^{9}$

Although there appears to be evidence for a benefit from previous work, ${ }^{6,7}$ accurately determining the magnitude of a differential association by PIK3CA status has implications for clinical trial design. The failure to demonstrate a significant interaction in both the meta-regression of previous observational studies ${ }^{23}$ and the present cohort would suggest that caution is required before considering additional randomized clinical trials that only recruit patients with mutated PIK3CA tumors (NCT02647099 and NCT02467582). On this basis, planned PTGS2 and PIK3CA subgroup analysis of trials evaluating adjuvant aspirin use in colorectal cancer patients, irrespective of tumor molecular profile, is perhaps more appropriate.

A major strength of this study is the inclusion of population-representative colon cancer patients. Furthermore, application of a precise, automated and validated digital immunoscoring system ensures reproducible immunoexpression data. However, there are a number of limitations that warrant further discussion. Most importantly, information on aspirin prescription was only assessed in the early post-operative period and no information was available regarding the duration of previous exposure. Assessment at this single time point may not reflect changes in postdiagnostic use and could lead to misclassification bias. ${ }^{23,25}$ However, the optimal duration and timing of aspirin exposure is not clear from observational data to date. It is possible that the important intervention period for aspirin use is in the early post-operative period, as seen for chemotherapy, ${ }^{26,27}$ in which case our data would capture the optimal aspirin time point. Over the counter medication use is another source of bias in pharmacoepidemiology studies, however medication records in COIS and the medical charts relied on a doctor patient interaction, therefore significant over the counter use is likely to have been captured. Also, previous pharmacoepidemiology studies have confirmed that between 75 and $98 \%$ of chronic low-dose aspirin use in the age-group of patients included in this cohort in the UK was by prescription. $^{28,29}$

The ColoCarta panel also only identifies $79 \%$ of known PIK3CA mutations. However, these hotspot mutations are representative of $84 \%$ of the exon 9 and 20 mutations detected in the seminal paper by Liao et al. ${ }^{6}$ It is therefore unlikely that detection of less common mutations would alter the PIK3CA stratified results as the number of additional aspirin users assigned as having a PIK3CA-mutant tumor would be small. Finally, we were unable to adjust for a number of confounding factors such as physical inactivity and obesity. ${ }^{30-32}$ However, to the best of the authors' knowledge, there is no evidence to suggest these factors would impact analyses stratified by PTGS2 or PIK3CA status.

Caution should be exercised when considering the association between better survival and aspirin use in the PTGS2-high subgroup given the borderline significance and multiple hypotheses tested. However, in sensitivity analyses the relationship appeared to be independent of statin use, a further medication with potential anti-cancer effects, ${ }^{33}$ and became more significant when the threshold PTGS2 H-score cutpoint was increased. Statistical power to detect an interaction by PIK3CA mutation status was also limited. However, the numbers were similar to the study by Domingo et al. $^{7}$ who only had 14 aspirin users in the PIK3CA-mutant group; the only difference being they had no events in this group whereas five events were observed among 17 patients in our cohort. 
In summary, aspirin use was associated with better survival in this population-based colon cancer cohort study and the association was more pronounced in tumors with higher levels of PTGS2 expression. In contrast, no significant interaction was observed with PIK3CA mutation status therefore caution should be exercised when considering trials assessing aspirin use in PIK3CA mutated tumors only. Planned subgroup analysis of trials evaluating adjuvant aspirin therapy in colorectal cancer patients, irrespective of tumor molecular profile, may therefore be more appropriate.

\section{CONFLICT OF INTEREST}

Guarantor of the article: Ronan T. Gray, MB BCh (Hons), MSc, MRCS.

Specific author contributions: Study conception and design: Marie M. Cantwell, Helen G. Coleman, Maurice B. Loughrey, Stephen McQuaid, Chris R. Cardwell, Jacqueline A. James, Brian T. Johnston, Manuel Salto-Tellez, and Liam J. Murray; Data acquisition: Ronan T. Gray, Helen G. Coleman, Maurice B. Loughrey, Peter Bankhead, Stephen McQuaid, Roisin F O'Neill, Kenneth Arthur, Victoria Bingham, Claire McGready, and Jacqueline A. James; Data analysis and interpretation: Ronan T. Gray, Helen G. Coleman, Chris R. Cardwell, and Liam J. Murray; Drafting manuscript: Ronan T. Gray, Helen G. Coleman, Stephen McQuaid, Chris R. Cardwell and Liam J. Murray. Manuscript revision: All. Final approval: All.

Financial support: This project was supported by a Health and Social Care (HSC) Research \& Development Division of the Public Health Agency Doctoral Fellowship (EAT/4905/13 -RTG), a Cancer Research UK (CRUK) Research Bursary (C50104/A17592-RTG), and a CRUK Population Health Postdoctoral Fellowship (C37703/A15333-H.G.C.). H.G.C., M.M.C., L.J.M., R.T.G. and R.F.O'N are all co-investigators or affiliated members of the UKCRC Centre of Excellence for Public Health Northern Ireland. The Northern Ireland Cancer Registry is funded by the Public Health Agency, Northern Ireland. The Northern Ireland Biobank is funded by the HSC Research and Development Division of the Public Health Agency in Northern Ireland and CRUK through the Belfast CRUK Centre, the Northern Ireland Experimental Cancer Medicine Centre and Friends of the Cancer Centre. The Northern Ireland Molecular Pathology Laboratory has received funding from CRUK, the Friends of the Cancer Centre, and the Sean Crummey Foundation. The study funders played no role in the study design or collection, analysis, and interpretation of data.

Potential competing interests: Peter W. Hamilton is Founder and Director in PathXL Ltd. Manuel Salto-Tellez is a senior advisor to PathXL. The remaining authors declare no conflict of interest.

Acknowledgments. We acknowledge the staff of the Northern Ireland Cancer Registry who facilitated data access to establish this cohort and Professor Brendan Pang (visiting professor Northern Ireland Molecular Pathology Laboratory) for a significant contribution to MSI analysis.

\section{Study Highlights}

\section{WHAT IS CURRENT KNOWLEDGE}

$\checkmark$ Epidemiological studies suggest aspirin use may be associated with improved survival after colorectal cancer diagnosis.

$\checkmark$ This association may vary according to molecular markers of phosphatidylinositol 3-kinase (PI3K) signaling activity.

\section{WHAT IS NEW HERE}

$\checkmark$ Aspirin use was associated with improved colorectal cancer-specific survival.

$\checkmark$ The association with mortality differed according to PTGS2 expression but not PIK3CA mutation status.

\section{TRANSLATIONAL IMPACT}

$\checkmark$ Limiting adjuvant aspirin trials to PIK3CA-mutant colorectal cancer may be too restrictive.

1. Algra AM, Rothwell PM. Effects of regular aspirin on long-term cancer incidence and metastasis: a systematic comparison of evidence from observational studies versus randomised trials. Lancet Oncol 2012; 13: 518-527.

2. Rothwell PM, Wilson M, Price JF et al. Effect of daily aspirin on risk of cancer metastasis: a study of incident cancers during randomised controlled trials. Lancet 2012; 379: 1591-1601.

3. Bains SJ, Mahic M, Myklebust TA et al. Aspirin as secondary prevention in patients with colorectal cancer: an unselected population-based study. J Clin Oncol 2016; 34: 2501-2508.

4. Elwood PC, Gallagher AM, Duthie GG et al. Aspirin, salicylates, and cancer. Lancet 2009; 373: $1301-1309$.

5. Ogino S, Lochhead P, Giovannucci E et al. Discovery of colorectal cancer PIK3CA mutation as potential predictive biomarker: power and promise of molecular pathological epidemiology. Oncogene 2014; 33: 2949-2955.

6. Liao X, Lochhead P, Nishihara R et al. Aspirin use, tumor PIK3CA mutation, and colorectalcancer survival. N Engl J Med 2012; 367: 1596-1606.

7. Domingo $\mathrm{E}$, Church DN, Sieber $\mathrm{O}$ et al. Evaluation of PIK3CA mutation as a predictor of benefit from nonsteroidal anti-inflammatory drug therapy in colorectal cancer. J Clin Oncol 2013; 31: 4297-4305.

8. Ogino S, Chan AT, Fuchs CS et al. Molecular pathological epidemiology of colorectal neoplasia: an emerging transdisciplinary and interdisciplinary field. Gut 2011; 60: 397-411.

9. Chan AT, Ogino S, Fuchs CS. Aspirin use and survival after diagnosis of colorectal cancer. JAMA 2009; 302: 649-659.

10. Yuan TL, Cantley LC. PI3K pathway alterations in cancer: variations on a theme. Oncogene 2008; 27: 5497-5510.

11. Kaur J, Sanyal SN. PI3-kinase/Wnt association mediates COX-2/PGE2 pathway to inhibit apoptosis in early stages of colon carcinogenesis: chemoprevention by diclofenac. Tumor Biol 2010; 31: 623-631.

12. Uddin $S$, Ahmed M, Hussain A et al. Cyclooxygenase-2 inhibition inhibits PI3K/AKT kinase activity in epithelial ovarian cancer. Int J Cancer 2010; 126: 382-394.

13. Kothari N, Kim R, Jorissen RN et al. Impact of regular aspirin use on overall and cancerspecific survival in patients with colorectal cancer harboring a PIK3CA mutation. Acta Oncol 2015; 54: 487-492.

14. Reimers MS, Bastiaannet $E$, Langley RE et al. Expression of HLA class I antigen, aspirin use, and survival after a diagnosis of colon cancer. JAMA Intern Med 2014; 174: 732-739.

15. Cardwell CR, Kunzmann AT, Cantwell MM et al. Low-dose aspirin use after diagnosis of colorectal cancer does not increase survival: a case-control analysis of a populationbased cohort. Gastroenterology 2014; 146: 700-708.

16. Zhang D, Salto-Tellez M, Putti TC et al. Reliability of tissue microarrays in detecting protein expression and gene amplification in breast cancer. Mod Pathol 2003; 16: 79-84.

17. Boyle DP, McArt DG, Irwin $G$ et al. The prognostic significance of the aberrant extremes of p53 immunophenotypes in breast cancer. Histopathology 2014; 65: 340-352.

18. McCarty KS, Szabo E, Flowers JL et al. Use of a monoclonal anti-estrogen receptor antibody in the immunohistochemical evaluation of human tumors. Cancer Res 1986; 46: 4244s-4248s.

19. Budczies J, Klauschen F, Sinn BV et al. Cutoff Finder: a comprehensive and straightforward web application enabling rapid biomarker cutoff optimization. PLOS ONE 2012; 7: e51862.

20. Fumagalli D, Gavin PG, Taniyama $Y$ et al. A rapid, sensitive, reproducible and cost-effective method for mutation profiling of colon cancer and metastatic lymph nodes. BMC Cancer 2010; 10: 101.

21. Gavin PG, Colangelo LH, Fumagalli $D$ et al. Mutation profiling and microsatellite instability in stage II and III colon cancer: an assessment of their prognostic and oxaliplatin predictive value. Clin Cancer Res 2012; 18: 6531-6541.

22. Khan NF, Perera R, Harper S et al. Adaptation and validation of the Charlson Index for Read/ OXMIS coded databases. BMC Fam Pract 2010; 11: 1. 
23. Paleari L, Puntoni M, Clavarezza M et al. PIK3CA mutation, aspirin use after diagnosis and survival of colorectal cancer. A systematic review and meta-analysis of epidemiological studies. Clin Oncol 2016; 28: 317-326.

24. Elwood PC, Morgan G, Pickering JE et al. Aspirin in the treatment of cancer: reductions in metastatic spread and in mortality: a systematic review and meta-analyses of published studies. PLOS ONE 2016; 11: e0152402.

25. Lévesque LE, Hanley JA, Kezouh A et al. Problem of immortal time bias in cohort studies: example using statins for preventing progression of diabetes. BMJ 2010; 340: b5087.

26. Biagi JJ, Raphael MJ, Mackillop WJ et al. Association between time to initiation of adjuvant chemotherapy and survival in colorectal cancer: a systematic review and meta-analysis. JAMA 2011; 305: 2335-2342.

27. Bos ACRK, van Erning FN, van Gestel YRBM et al. Timing of adjuvant chemotherapy and its relation to survival among patients with stage III colon cancer. Eur J Cancer 2015; 51 2553-2561.

28. Yang $\mathrm{Y}-\mathrm{X}$, Hennessy $\mathrm{S}$, Propert $\mathrm{K}$ et al. Chronic statin therapy and the risk of colorectal cancer. Pharmacoepidemiol Drug Saf 2008; 17: 869-876.

29. Bedson J, Whitehurst T, Lewis $M$ et al. Factors affecting over-the-counter use of aspirin in the secondary prophylaxis of cardiovascular disease. Br J Gen Pract 2001; 51: 1001-1003.

30. Baade PD, Meng X, Youl PH et al. The impact of body mass index and physical activity on mortality among patients with colorectal cancer in Queensland, Australia. Cancer Epidemio Biomarkers Prev 2011; 20: 1410-1420.

31. Haydon AMM, Maclnnis RJ, English DR et al. Effect of physical activity and body size on survival after diagnosis with colorectal cancer. Gut 2006; 55: 62-67.
32. Kuiper JG, Phipps Al, Neuhouser ML et al. Recreational physical activity, body mass index, and survival in women with colorectal cancer. Cancer Causes Control 2012; 23: 1939-1948.

33. Thurnher M, Nussbaumer O, Gruenbacher G. Novel aspects of mevalonate pathway inhibitors as antitumor agents. Clin Cancer Res 2012; 18: 3524-3531.

Clinical and Translational Gastroenterology is an openaccess journal published by Nature Publishing Group. This work is licensed under a Creative Commons AttributionNonCommercial-ShareAlike 4.0 International License. The images or other third party material in this article are included in the article's Creative Commons license, unless indicated otherwise in the credit line; if the material is not included under the Creative Commons license, users will need to obtain permission from the license holder to reproduce the material. To view a copy of this license, visit http:/l creativecommons.org/licenses/by-nc-sa/4.0/

(C) The Author(s) 2017 